\title{
Physiological and Morphological Characterization of Organotypic Cocultures of the Chick Forebrain Area MNH and its Main Input Area DMA/DMP
}

\author{
Heike Endepols, ${ }^{1 \S}$ Julia Jungnickel $^{1,2}$ and Katharina Braun ${ }^{1,2}$ \\ ${ }^{1}$ Leibniz-Institute for Neurobiology, Brenneckestr. 6, 39118 Magdeburg, Germany \\ ${ }^{2}$ Otto-von-Guericke University Magdeburg, Inst. for Biology, Dept. Zoology/ \\ Developmental Neurobiology, Brenneckestr. 6, 39118 Magdeburg, Germany
}

\begin{abstract}
SUMMARY
Cocultures of the learning-relevant forebrain region mediorostral neostriatum and hyperstriatum ventrale (MNH) and its main glutamatergic input area nucleus dorsomedialis anterior thalami/posterior thalami were morphologically and physiologically characterized. Synaptic contacts of thalamic fibers were lightand electron-microscopically detected on MNH neurons by applying the fluorescence tracer DiI-C18(3) into the thalamus part of the coculture. Most thalamic synapses on MNH neurons were symmetric and located on dendritic shafts, but no correlation between Gray-type ultrastructure and dendritic localization was found. Using intracellular current clamp recordings, we found that the electrophysiological properties, such as input resistance, time constant, action potential threshold, amplitude, and duration of MNH neurons, remain stable for over 30 days in
\end{abstract}

Reprint requests to: Katharina Braun, Otto-von-Guericke University Magdeburg, Institute for Biology, Department of Zoology/Developmental Neurobiology, Brenneckestr. 6, 39118 Magdeburg. Germany, e-mail: braun@ifn-magdeburg.de

\$Present address: Institute of Zoology, University of Cologne, Weyertal 119, 50923 Köln. Germany vitro. Pharmacological blockade experiments revealed glutamate as the main neurotransmitter of thalamic synapses on MNH neurons, which were also found on inhibitory neurons. High frequency stimulation of thalamic inputs evoked synaptic potentiation in $22 \%$ of $\mathrm{MNH}$ neurons. The results indicate that DMA/DMP-MNH cocultures, which can be maintained under stable conditions for at least 4 weeks, provide an attractive in vitro model for investigating synaptic plasticity in the avian brain.

\section{KEYWORDS}

electron microscopy, electrophysiology, synaptic plasticity, glutamate, GABA

\section{INTRODUCTION}

Avian learning-for example, song learning in song birds (Mooney, 1999; Bischof \& Rollenhagen, 1999; Tramontin \& Brenowitz, 2000), food storage and retrieval in jays (Griffiths et al., 1999), one-trial avoidance learning (Rose, 2000) and filial imprinting in domestic chicks (Bolhuis \& Honey, 1998; Horn, 1990; Braun et al., 1999)-is becoming 
increasingly popular as a model for studying the cellular and molecular mechanisms of vertebrate learning and memory formation. The systematic analysis of filial imprinting, representing the very first postnatal learning event in birds and in various mammalian species, including humans (DeCasper \& Fifer, 1980), has lead to the identification of morphological, biochemical, molecular, and physiolological changes accompanying this ontogenetically driven form of emotional learning. The presumed avian analogue of the prefrontal cortex (Metzger et al., 1996; Scheich et al., 1991; Bock et al., 1997), the mediorostral neostriatum and hyperstriatum ventrale (MNH), is one key structure in the chick forebrain that is critically involved in auditory filial imprinting.

Increased metabolic activity (Maier \& Scheich, 1983; Bock et al., 1996; Wallhäußer \& Scheich, 1987), spike activity (Bredenkötter \& Braun, 1997; 2000), glutamate release (Gruss \& Braun, 1996), and a $45 \%$ to $50 \%$ reduction of spine synapses (Wallhäußer \& Scheich, 1987; Bock \& Braun, 1998) were seen in the MNH after auditory imprinting. Behavioral, morphological, and in vivo microdialysis studies revealed that glutamate, released by afferents arising from the nucleus dorsomedialis anterior thalami/posterior thalami (DMA/DMP) (Metzger et al., 1996), is essential for this learning task. In particular, the activation of the $\mathrm{N}$-methyl-D-aspartate (NMDA) receptor mediates the induction of long-term potentiation (LTP) and long-term depression (LTD) in MNH neurons (Wang et al., 1994), as well as the learning-induced spine elimination (Bock et al., 1996; Bock \& Braun, 1999).

To analyze the physiological properties of the glutamatergic afferents on $\mathrm{MNH}$ neurons on the single cell level, we developed an in vitro preparation, 'organotypic' cocultures of DMA/DMP and $\mathrm{MNH}$, in which well-controllable pharmacological and electrical stimulation experiments can be done under stable conditions (Hofmann \& Braun, 1995).

\section{EXPERIMENTAL}

\section{Preparation of slice cultures}

The preparation of MNH slices was described elsewhere (Hofmann \& Braun, 1995). Briefly, on the day of hatching, White Leghorn chicks (Gallus gallus domesticus) were decapitated and the forebrain was embedded in sterile agarose (LMP-agarose, Bethesda Research Lab., $2 \%$ in distilled water) and glued to the cutting block of a vibratome. Transverse $250 \mu \mathrm{m}$ slices were sectioned into fresh icecold dissecting buffer (Geys Balanced Salt Solution, Gibco) enriched with glucose $(5 \mathrm{mg} / \mathrm{ml})$, and the neostriatal part of MNH was dissected out with a scalpel (Fig. 1A). The MNH slices were kept in dissecting buffer for 90 to $120 \mathrm{~min}$ at $4^{\circ} \mathrm{C}$.

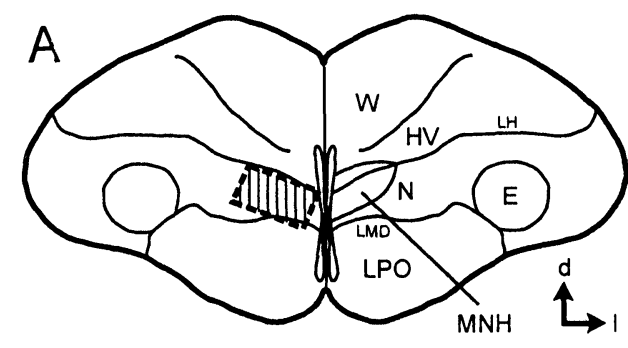

B

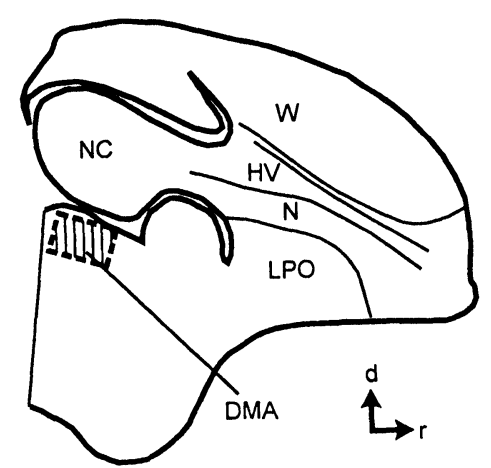

Fig. 1: Schematic drawing of a transverse (A) and a sagittal section (B) of a domestic chick brain. Hatched regions indicate the slices used for cocultures. Abbreviations: $\mathrm{d}$ : dorsal; DMA: nucleus dorsomedialis anterior thalami; E: ectostriatum; HV: hyperstriatum ventrale; l: lateral; LH: lamina hyperstriatica; LMD: lamina medullaris dorsalis; LPO: lobus parolfactorius; $\mathrm{MNH}$ : mediorostral neostriatum and hyperstriatum ventrale; $\mathrm{N}$ : neostriatum; $\mathrm{NC}$ : neostriatum caudale; r: rostral; W: Wulst. 
The optimal age for thalamic explants was determined in pilot studies, which revealed the best results (judged by survival of cultures and ingrowth of fibers from thalamus to $\mathrm{MNH}$ ) for 14-dold chick embryos (Fig. 1B). For slice preparation, the entire brain was removed, split into two halves, and embedded in $40^{\circ} \mathrm{C}$ sterile agarose. The two agarose blocks containing the two hemispheres were glued side by side onto the cutting block of a vibratome, with the midline oriented upward, and $250 \mu \mathrm{m}$ parasagittal sections were made. Under microscopic control, the DMA/DMP was cut out with a scalpel while using the habenula, which is separated from DMA/DMP by a sharp borderline, as a landmark. DMA/DMP slices were maintained for 30 to $60 \mathrm{~min}$ in dissecting buffer at $4^{\circ} \mathrm{C}$.

One MNH slice was placed next to one DMA/ DMP slice (maximal distance $1 \mathrm{~mm}$ ) onto a coverslip $(12 \times 24 \mathrm{~mm})$ and embedded into a plasma clot consisting of two drops of chicken plasma (Cocalico) and one drop of thrombin (Sigma). The coverslips were inserted into $10 \mathrm{ml}$ plastic culture tubes (Nunc) with $0.5 \mathrm{ml}$ medium and placed into a roller drum that rotated at approximately 20 revolutions $/ \mathrm{h}$. The cultures were incubated at $37^{\circ} \mathrm{C}$ in dry air.

The culture medium consisted of $50 \%$ Basal medium (Eagle; Gibco), with phenol red, $\mathrm{pH}$ 7.2, 25\% Earls Balanced Salt Solution (Gibco), and $25 \%$ horse serum (Gibco), supplemented with D-glucose to a final concentration of $5 \mathrm{rng} / \mathrm{ml}$. The horse serum was heat inactivated for $30 \mathrm{~min}$ at $56^{\circ} \mathrm{C}$.

On the first day in vitro (DIV) a cocktail of mitotic inhibitors was added to the medium for $24 \mathrm{~h}$ to prevent excessive glia proliferation. The three components uridine, cytosine- $\beta$ - $D$-arabinofuranoside and 5-fluorodeoxyuridine (all from Sigma) had a final concentration of $0.1 \mu \mathrm{M}$. From the second DIV, medium without mitotic inhibitor was used and replaced twice a week.

\section{Tracing the DMA/DMP-MNH projection with DiI}

Cultures of different ages (6 to 28 DIV) were fixed for $30 \mathrm{~min}$ in $2 \%$ paraformaldehyde in cacodylate buffer $(0.1 \mathrm{M}, \mathrm{pH} 7.3)$ in culture tubes at $4{ }^{\circ} \mathrm{C}$. A crystal of the fluorescent tracer DiI-C18(3) (1,1'-dioctadecyl-3,3,3',3'-tetramethylindocarbocyanine perchlorate; Molecular Probes) was placed on the DMA/DMP part of the coculture and then incubated for 2 to $3 \mathrm{~d}$ at $37^{\circ} \mathrm{C}$. The progress of Dil tracing along the DMA/DMP axons was monitored daily.

Photoconversion of DiI: In four cultures (14 DIV) DiI was photoconverted into an electrondense DAB reaction product for electron microscopic observation. Cultures were incubated for 1 $\mathrm{h}$ in $2 \mathrm{mg} / \mathrm{ml} \mathrm{DAB}$ in Tris buffer $\mathrm{pH} 8.4$ and then exposed to fluorescence light $(550 \mathrm{~nm}, 20 \mathrm{x}$ objective). Quality of staining was observed during the photoconversion procedure. The cultures were removed from the coverslips, and a piece of the MNH part $\left(0.5\right.$ to $\left.1 \mathrm{~mm}^{2}\right)$ with dense thalamic innervation was dissected out. The tissue was washed for $30 \mathrm{~min}$ in cacodylate buffer, postfixed for $30 \mathrm{~min}$ in $2 \%$ glutaraldehyde in cacodylate buffer at $4^{\circ} \mathrm{C}$, and then incubated for $30 \mathrm{~min}$ in $1 \% \mathrm{OsO}_{4}$ in cacodylate buffer at RT. After several washes in $70 \%$ ethanol, the tissue was block-contrasted for $30 \mathrm{~min}$ in $70 \%$ ethanol saturated with uranyl acetate. Following dehydration in ethanol solutions $(80 \%$. $90 \%, 96 \%, 2 \times 100 \%, 15$ min each), the block was embedded for 3 days in Low Viscosity Spurr Kit (Pelco) at $60^{\circ} \mathrm{C}$. Ultrathin $(80 \mathrm{~nm})$ sections horizontal to the surface of the cultured tissue were collected on slot grids coated with Formvar film and examined with a Zeiss EM 902A electron microscope. Photographs of synapses were taken at a magnification of $20,000 x$ or $30,000 x$. The negatives were digitized with the help of a scanner (Arcus, Agfa), and postsynaptic density (PSD) length and presynaptic area of each synapse was measured using a Macintosh 7100/66 (software NIH Image). 
Additionally, we determined the presence of presynaptic mitochondria, synaptic curvature, localization of the presynapse (spine, dendrite, axon or soma), Gray-type of the synapse (symmetric or asymmetric), percentage of perforated synapses (synapses with a discontinuous PSD), and percentage of multiple synapses (i.e. a presynaptic terminal contacts more than one postsynaptic element).

\section{Electrophysiology}

Electrophysiological experiments were carried out in a $1 \mathrm{ml}$ recording chamber with a glass bottom, mounted on an inverse microscope (Zeiss Axiovert $35 \mathrm{M})$. During recording, cultures were submerged in $36^{\circ} \mathrm{C}$ artificial cerebrospinal fluid (ACSF), consisting of (mM): $\mathrm{NaCl}, 121 ; \mathrm{KCl}, 3$; $\mathrm{MgSO}_{4}, 2.4 ; \mathrm{NaHCO}_{3}, 23 ; \mathrm{CaCl}_{2}, 2.5 ; \mathrm{KH}_{2} \mathrm{PO}_{4}, 1$; glucose, 12. The ACSF was aerated with carbogen $\left(95 \% \mathrm{O}_{2} / 5 \% \mathrm{CO}_{2}\right)$; the flow rate was $0.3 \mathrm{ml} / \mathrm{min}$.

Recording electrodes were pulled from glass pipettes (Hilgenberg) and filled with $1 \mathrm{M}$ potassium acetate solution, $\mathrm{pH}$ 7.4. The D.C. resistance was 80 to $150 \mathrm{M} \Omega$. Intracellular recordings of neurons were performed in the $\mathrm{MNH}$ part of the coculture using an amplifier (Axoclamp-2A, Axon Instruments) in bridge mode. The stimulation electrode, a monopolar tungsten electrode insulated with pacemaker varnish, was placed into the DMA/DMP part of the coculture. After stabilization of the intraceilular recording, DMA/DMP neurons were stimulated using single biphasic test pulses $(0.1 \mathrm{~ms}, 5$ $25 \mathrm{~V})$ or high frequency stimulus trains $(0.1 \mathrm{~ms}, 5-$ $25 \mathrm{~V}, 100 \mathrm{~Hz}, 1 \mathrm{~s})$, delivered by a stimulus isolator (WPI). Intracellular signals were displayed on a digitizing oscilloscope (Gould), and evoked responses were stored with a data recording system (TIDA, Heka-Electronics) for further analyses.

In pharmacological experiments, the following substances were injected into the supply hose of the recording chamber:
- NMDA (N-methyl-D-aspartate; Sigma), glutamate agonist

- APV (DL-2-amino-5-phosphonovaleric acid; RBI), NMDA antagonist

- Con-G (Conantokin-G; gift from Dr. J.L. Torres), NMDA antagonist

- CNQX (6-cyano-7-nitroquinoxaline-2,3-dione; water-soluble complex with 2-hydroxypropyl$\beta$-cyclodextrin; RBI), AMPA/kainate antagonist

- (-)-Bicuculline methiodid (Sigma), GABA-A antagonist

\section{Data analysis}

General electrophysiological properties were investigated in cultures of the following ages: 5 to 9 DIV, 12 to 16 DIV, 19 to 23 DIV, and 26 to 30 DIV. The values of the four groups were compared using the Kruskal-Wallis ANOVA on Ranks. The resting membrane potential was estimated from the difference between the intracellularly recorded membrane potential and the extracellular potential upon withdrawal from the cell. Input resistance was determined from the slope of the linear range of the steady-state current-voltage relationship near the resting membrane potential. The time constant was calculated as the time to reach $63 \%$ of the steady-state response to a hyperpolarizing intracellular current pulse $(-0.2$ or $-0.3 \mathrm{nA})$. The action potential (AP) amplitude and duration were determined using synaptically evoked APs. The AP amplitude was measured from resting potential level to peak. The AP duration was measured where the action potential was half its peak amplitude ('half-width').

In potentiation experiments, we analyzed the responses to single-test stimuli (applied with $0.5 \mathrm{~Hz}$ ) before and after high frequency stimulation $(100 \mathrm{~Hz}, 1 \mathrm{~s})$ : Mean values of EPSP slope, integral of EPSP, number of evoked APs and latency of evoked APs were calculated from 
trains of 25 single thalamic stimulations and analyzed using the Friedman Repeated Measures Analysis of Variance on Ranks, and Dunn's Method for Comparison of all Groups versus Control Group. For all statistical procedures, the $p$-values were set at $p \leq 0.05$.

\section{RESULTS}

\section{DiI tracing}

Light microscopic observation revealed a massive projection from DMA/DMP into $\mathrm{MNH}$ after 8 DIV, even when initially the explants did not touch each other. Thalamic axons appeared thin and varicose (Fig. 2). After extended incubation periods, some backfilled MNH neurons became visible, indicating that $\mathrm{MNH}$ neurons projected back into DMA/DMP.

At the electron microscopic level, numerous Dil labeled thalamic axons were observed, forming terminal or en passant synapses with $\mathrm{MNH}$ neurons (Fig. 3). Measurement of 46 DiI labeled thalamic synapses of 4 cocultures revealed the following parameters (for details see Table 1): Mean PSD length was $0.77 \pm 0.32 \mu \mathrm{m}$, mean presynaptic area was $1.41 \pm 0.98 \mu \mathrm{m}^{2}$. Shaft synapses had a mean PSD length of $0.60 \pm 0.20 \mu \mathrm{m}$. Most synaptic contacts were symmetric (52\%) and located on dendritic shafts (62\%) (Fig. 3B). Nevertheless, no significant correlation was found between Gray-type and localization of the synapses (Spearman Rank Order Correlation). Spines bore symmetric as well as asymmetric thalamic synapses and so did dendritic shafts. Only $11 \%$ of all thalamus-MNH synapses were asymmetric and likewise located on dendritic spines.

Eleven percent of thalamic synapses displayed a perforated postsynaptic density, and $4.5 \%$ of presynapses contacted two postsynaptic structures (multiple synapses). Thirty nine percent of synaptic boutons contained one or two mitochondria. The synaptic curvatures were flat $(39 \%)$, presynaptic concave $(26 \%)$, and presynaptic convex $(35 \%)$.

\section{Electrophysiology}

We recorded 120 neurons in the MNH part of DMA/DMP-MNH cocultures. All MNH neurons showed spontaneous IPSPs, as well as EPSPs which could reach threshold. In most neurons, periods of low activity and periods of high activity alternated. The high-activity phase could often be triggered by a test stimulus in DMA/DMP.

Intrinsic electrophysiological properties. The electrophysiological properties (resting potential, time constant, input resistance, spike threshold, spike amplitude, and spike duration) of $61 \mathrm{MNH}$ neurons in culture were measured. The mean values \pm standard deviation of the parameters are summarized in Table 2. The mean resting potential of the age group 19 to 23 DIV was significantly more positive $(-61 \mathrm{mV})$ as compared with the age groups 5 to 9 DIV $(-68 \mathrm{mV})$ and 26 to $30 \mathrm{DIV}(-66 \mathrm{mV})$. The other parameters did not differ significantly.

\section{Spike patterns}

MNH neurons in culture could be classified into three types, according to firing patterns generated during a depolarizing intracellular current pulse.

Seventy-five percent of the neurons showed regular spiking behavior (Fig. 4A), generating trains of action potentials with increasing inter-spike intervals. In most of the regular spiking neurons action potentials were followed by afterhyperpolarizations of medium duration (mAHPs), after which the membrane potential gradually rose toward threshold, due to the continued current injection.

Twelve percent of the neurons were classified as intrinsically bursting (Fig. 4B): As a response to a depolarizing current pulse, two or more action 


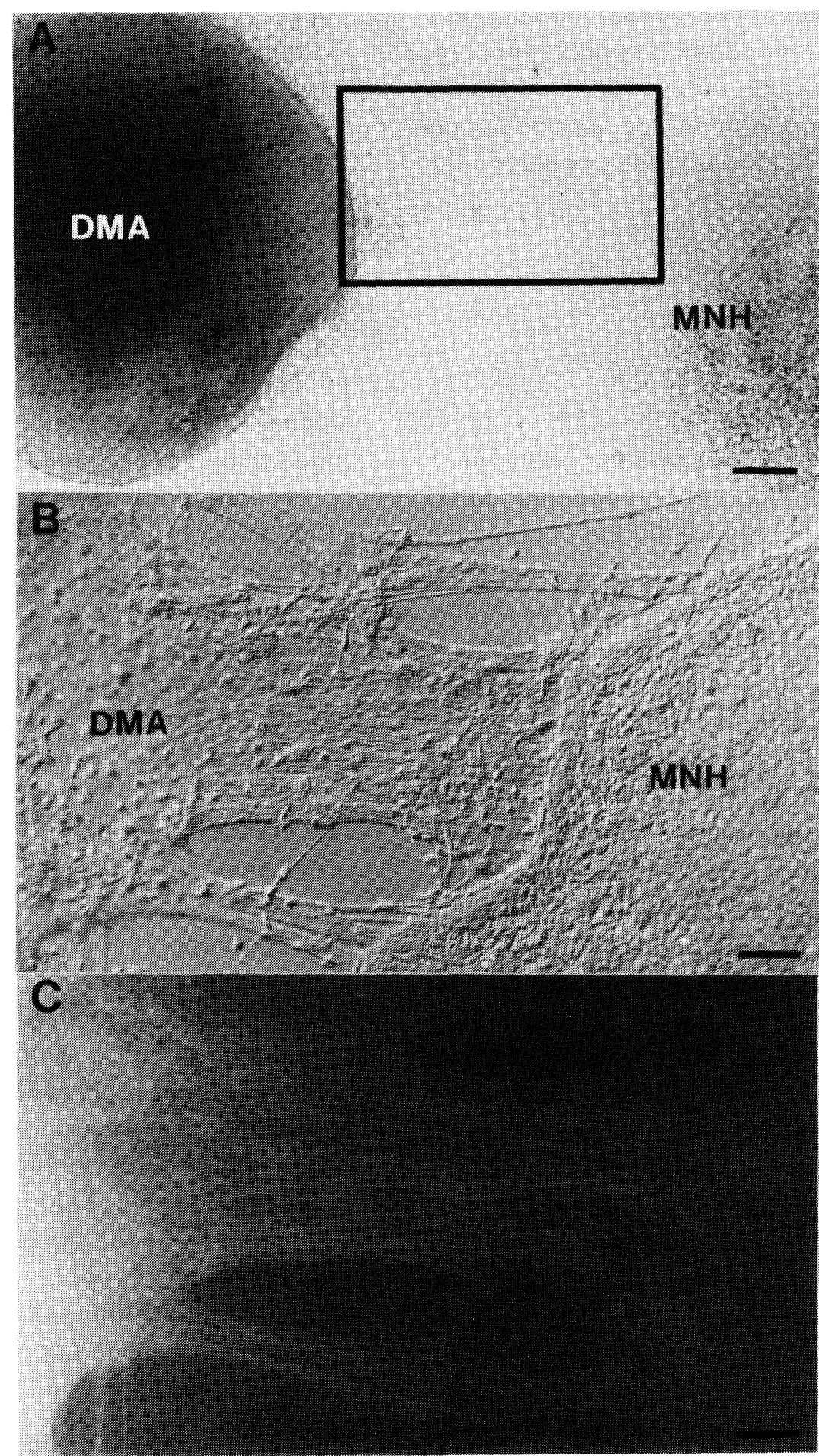

Fig. 2: Thalamus (DMA/DMP)-MNH coculture. A: Two Dil-crystals (asterisks) have been placed on the thalamus part of the coculture (DMA). B: Detail of the contact zone between the two explants (frame in A). C: Same detail as in B, fluorescence illumination. Numerous axons of thalamic neurons are visible which project in bundles into MNH. Scale bar: A: $250 \mu \mathrm{m}$, B+C: $100 \mu \mathrm{m}$. 

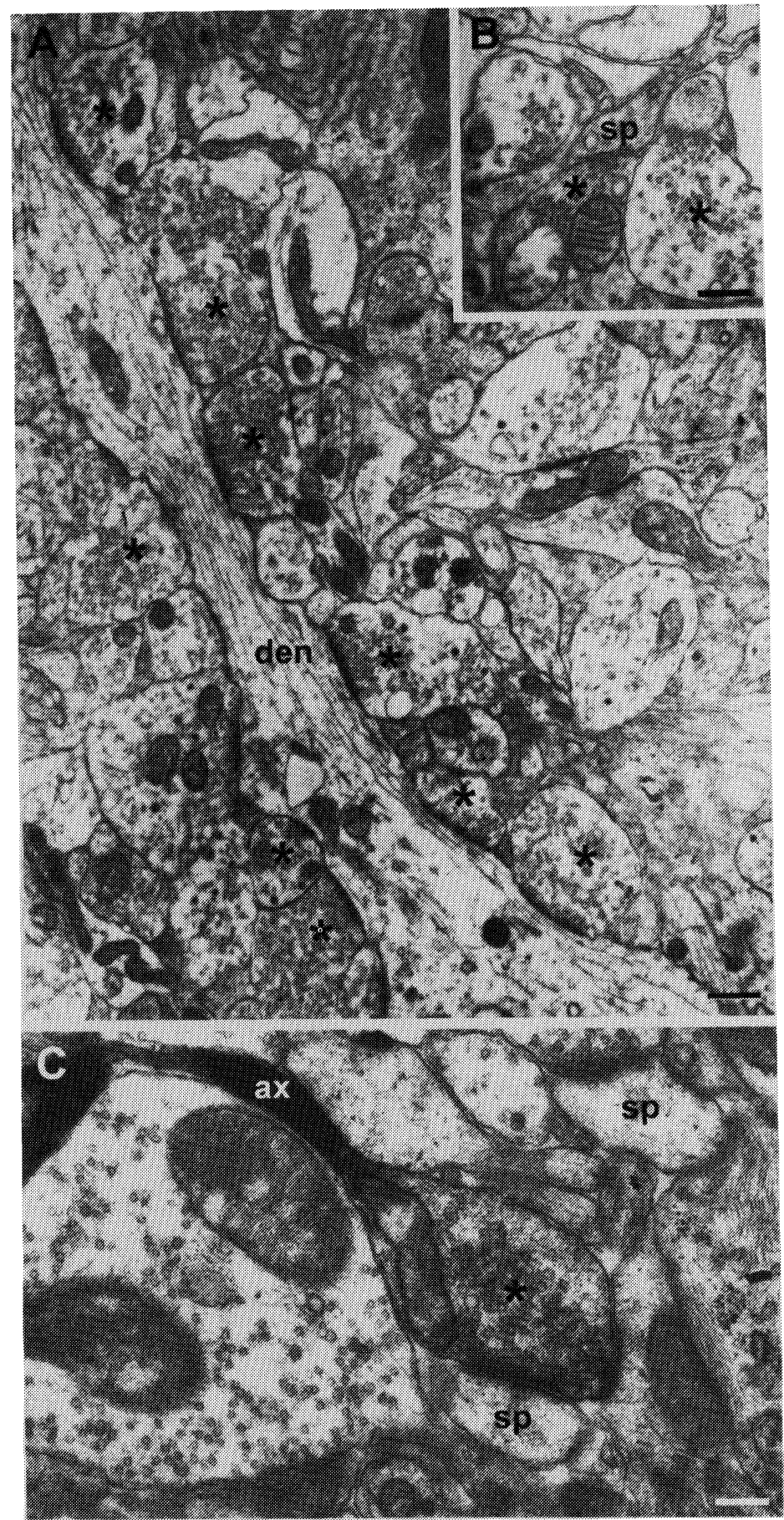

Fig. 3: A: MNH part of a coculture (14 DIV). A dendrite (den) of an $\mathrm{MNH}$ neuron is covered with numerous shaft synapses (asterisks). Scale bar: $1 \mu \mathrm{m}$. B: Example of a dendritic spine (sp) and two presynaptic terminals (asterisks). Scale bar: $500 \mathrm{~nm}$. C: Dil labeled thalamic axon terminal within the MNH part of the coculture (14 DIV). While the axon (ax) is stained by the electron dense product of Dil photoconversion, the synaptic bouton (asterisk) is not labeled and a presynaptic mitochondrion and synaptic vesicles are visible. The bouton contacts a dendritic spine (sp). Scale bar: $250 \mathrm{~nm}$. 
TABLE 1

Analysis of Dil labeled synaptic terminals in the MNH part of the coculture (14 DIV).

\begin{tabular}{|c|c|c|c|}
\hline parameter & all synapses & $\begin{array}{l}\text { spine synapses } \\
(34 \%)\end{array}$ & $\begin{array}{l}\text { shaft synapses } \\
(62 \%)\end{array}$ \\
\hline PSD length & $0.77 \pm 0.32 \mu \mathrm{m}$ & $0.60 \pm 0.21 \mu \mathrm{m}$ & $0.87 \pm 0.35 \mu \mathrm{m}$ \\
\hline presynaptic area & $1.41 \pm 0.98 \mu \mathrm{m}^{2}$ & $1.41 \pm 0.69 \mu \mathrm{m}^{2}$ & $1.14 \pm 0.98 \mu \mathrm{m}^{2}$ \\
\hline \multirow{2}{*}{ Gray-type of synapse } & $48 \%$ asymmetric & $33 \%$ asymmetric & $56 \%$ asymmetric \\
\hline & $52 \%$ symmetric & $67 \%$ symmetric & $44 \%$ symmetric \\
\hline \multirow{3}{*}{ synaptic curvature } & $39 \%$ flat & $53 \%$ flat & $37 \%$ flat \\
\hline & $26 \%$ presynaptic concave & $20 \%$ presynaptic concave & $30 \%$ presynaptic concave \\
\hline & $35 \%$ presynaptic convex & $27 \%$ presynaptic convex & $33 \%$ presynaptic convex \\
\hline perforated synapses & $11 \%$ & $13 \%$ & $11 \%$ \\
\hline multiple synapses & $4.5 \%$ & $20 \%$ & $0 \%$ \\
\hline $\begin{array}{l}\text { synapses with presynaptic } \\
\text { mitochondria }\end{array}$ & $39 \%$ & $27 \%$ & $59 \%$ \\
\hline $\mathrm{n}$ & $\begin{array}{l}46 \text { synapses out of } 4 \\
\text { cultures }\end{array}$ & $\begin{array}{l}27 \text { synapses out of } 4 \\
\text { cultures }\end{array}$ & $\begin{array}{l}15 \text { synapses out of } 4 \\
\text { cultures }\end{array}$ \\
\hline
\end{tabular}

TABLE 2

Electrophysiological properties of MNH neurons in culture

\begin{tabular}{cccccccc}
\hline age [DIV] & $\mathrm{n}$ & $\mathrm{RP}[\mathrm{mV}]$ & $\tau[\mathrm{ms}]$ & $\mathrm{R}_{\mathrm{N}}[\mathrm{M} \Omega]$ & $\mathrm{S}-\mathrm{Th}[\mathrm{mV}]$ & S-A [mV] & S-D [ms] \\
\hline $5-9$ & 11 & $-68 \pm 9$ & $6 \pm 3$ & $57 \pm 30$ & $-39 \pm 9$ & $65 \pm 11$ & $1.2 \pm 0.6$ \\
$12-16$ & 23 & $-61 \pm 6$ & $6 \pm 3$ & $53 \pm 23$ & $-46 \pm 7$ & $63 \pm 10$ & $1.3 \pm 0.4$ \\
$19-23$ & 15 & $-61 \pm 8 *$ & $5 \pm 3$ & $57 \pm 39$ & $-46 \pm 8$ & $60 \pm 13$ & $1.2 \pm 0.4$ \\
$26-30$ & 12 & $-66 \pm 7$ & $9 \pm 6$ & $67 \pm 12$ & $-51 \pm 7$ & $66 \pm 9$ & $1.1 \pm 0.4$ \\
\hline
\end{tabular}

$R P$, resting membrane potential; $\tau$, membrane time constant; $R_{N}$, input resistance; $S-T h$, spike threshold; S-A, spike amplitude; S-D, spike duration (half-width). Values represent mean \pm standard deviation. $* \mathrm{RP}$ of the age group 19-23 DIV was significantly more positive than RP of the age groups 5-9 DIV ( $p=0.035)$ and 26-30 DIV ( $p=0.043)$. 
A1

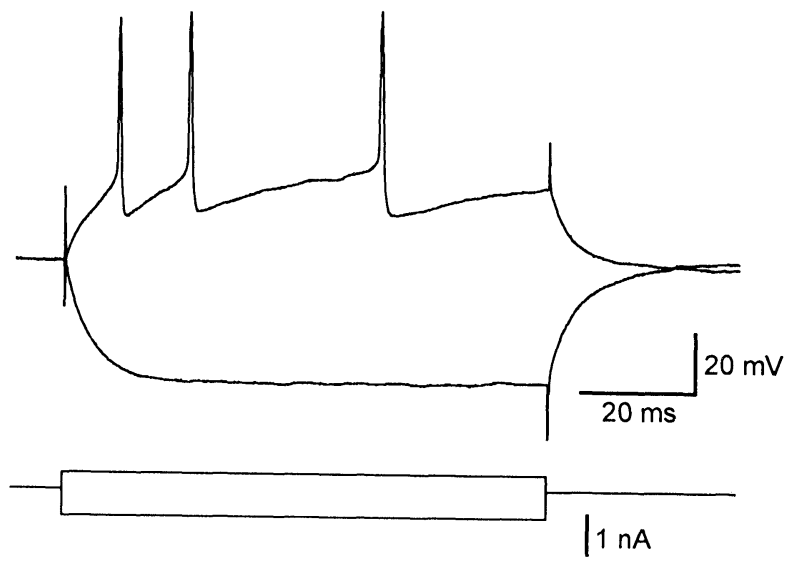

B1

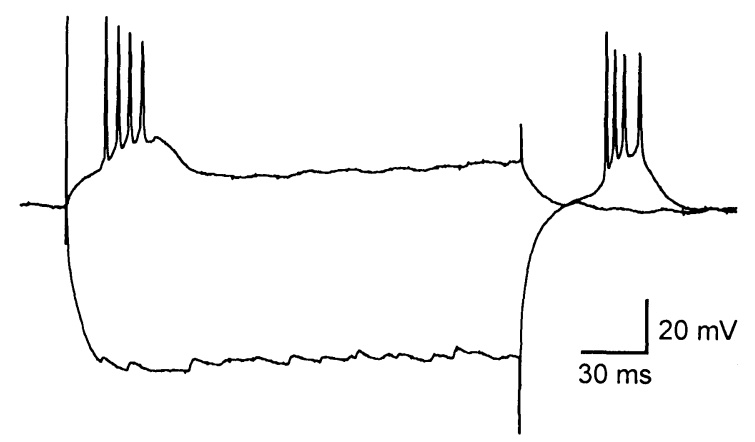

$11 \mathrm{nA}$
A2

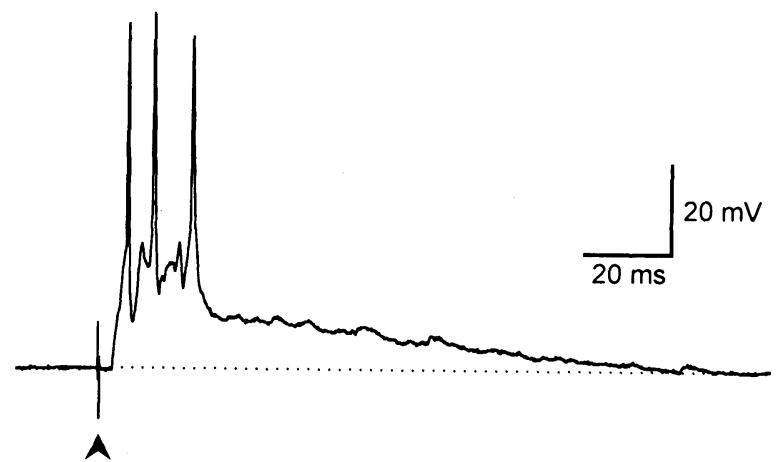

B2

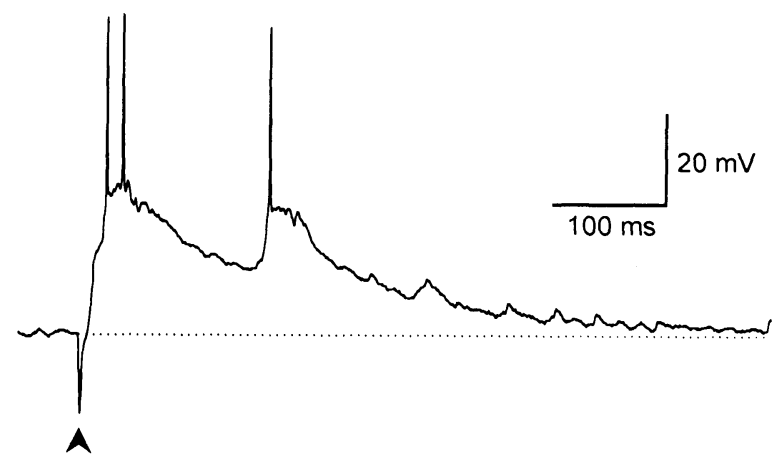

$\mathrm{C} 2$

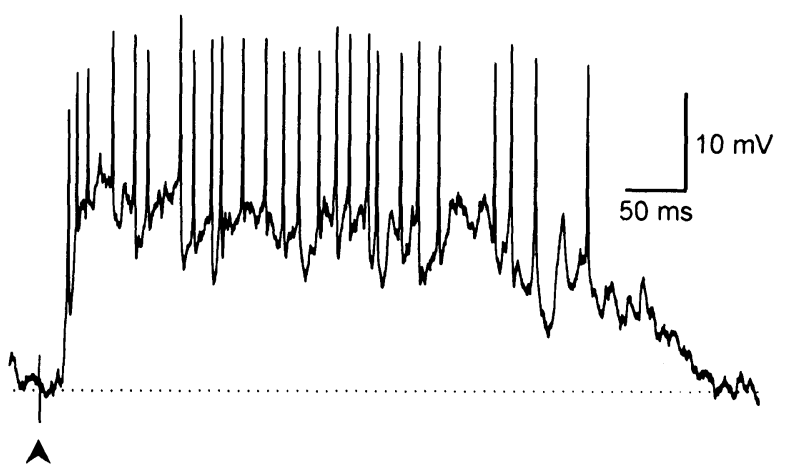

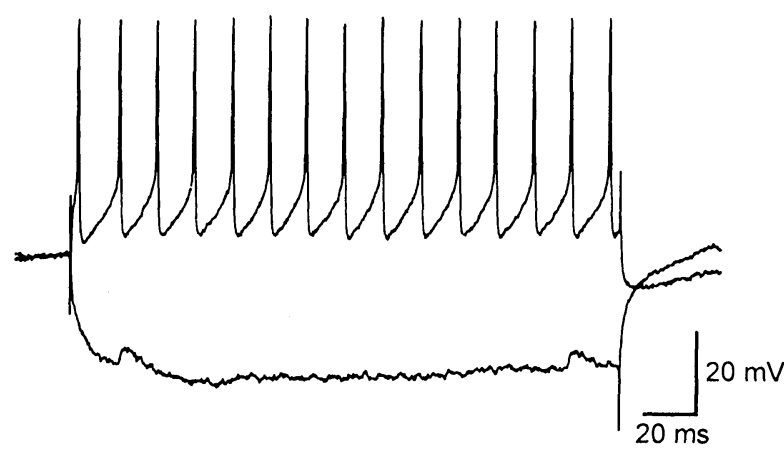

I $1 \mathrm{nA}$

Fig. 4: Electrophysiologically defined neuron types in the MNH part of the coculture. Column 1 shows the neuron's responses to intracellular current pulses after which the neuron is classified: the current steps are indicated below the recording traces. Column 2 illustrates the neuron's response to a single $100 \mu \mathrm{s}$ voltage pulse in the thalamus part of the coculture; the stimulus artifacts are indicated by arrowheads. A: Regular spiking neuron. B: Intrinsically bursting neuron. C: Fast spiking neuron. 
TABLE 3:

Electrophysiological properties of different neuron types of MNH in culture

\begin{tabular}{lccccccc}
\hline neuron type & $\mathrm{n}$ & $\mathrm{RP}[\mathrm{mV}]$ & $\tau[\mathrm{ms}]$ & $\mathrm{R}_{\mathrm{N}}[\mathrm{M} \Omega]$ & $\mathrm{S}-\mathrm{Th}[\mathrm{mV}]$ & $\mathrm{S}-\mathrm{A}[\mathrm{mV}]$ & $\mathrm{S}-\mathrm{D}[\mathrm{ms}]$ \\
\hline regular & 46 & $-63 \pm 8$ & $7 \pm 5$ & $59 \pm 32$ & $-46 \pm 8$ & $65 \pm 9$ & $1.3 \pm 0.4$ \\
fast & 8 & $-62 \pm 8$ & $4 \pm 2 *$ & $52 \pm 30$ & $-50 \pm 7$ & $53 \pm 13 *$ & $0.7 \pm 0.2 *$ \\
bursting & 7 & $-68 \pm 5$ & $5 \pm 1$ & $56 \pm 22$ & $-47 \pm 9$ & $66 \pm 11$ & $1.3 \pm 0.4$ \\
\hline
\end{tabular}

A

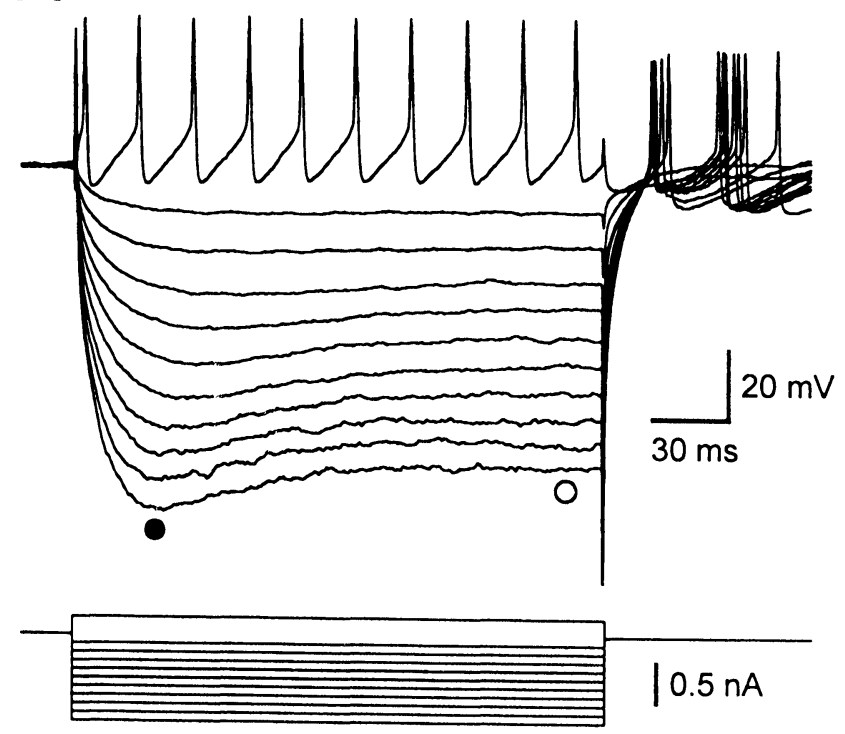

B

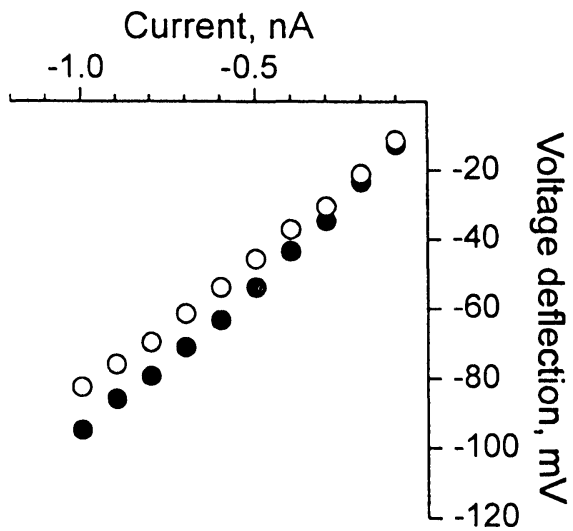

Fig. 5: Fast spiking MNH neuron with inward rectifier characteristics. A: Superimposed neuronal responses during one depolarizing and ten hyperpolarizing intracellular current pulses. An initial overshoot ("sag") of the membrane deflection at the beginning of the pulse is visible (filled circle). Current steps are indicated below. B: Current-voltage relationship. The amplitudes of membrane voltage deflections were measured at the beginning of the current pulse (filled circle) and at the end (open circle). The divergence of the two curves indicates the presence of an inward rectifier $\mathrm{K}^{+}$-channel. 
potentials were riding a depolarizing envelope. After the cessation of a hyperpolarizing current pulse, rebound bursts could often be observed. The bursts were followed by slow afterhyperpolarizations (sAHPs).

Thirteen percent of the neurons were fast spiking (Fig. 4C), showing minimal spike adaptation during prolonged depolarizing current pulses. Action potentials were followed by fast afterhyperpolarizations (fAHPs). In the fast spiking neurons, we observed a significantly lower amplitude and shorter duration of action potentials, as well as a shorter time constant than in bursting or regular spiking neurons. Other electrophysiological features were not significantly different. The electrophysiological properties of the three cell types are summarized in Table 3.

\section{Inward rectification}

Thirty-three percent of the MNH neurons showed nonlinearities within the electrotonic response to intracellular hyperpolarization. At negative current values an initial overshoot ('sag') in the response to hyperpolarizing current pulses occurred (Fig. 5A), and the slope of the currentvoltage relationship decreased (Fig. 5B). After the cessation of the hyperpolarizing pulse, anodal break overshoot of the membrane potential could exceed threshold and generate rebound action potentials. These findings indicate the presence of the inward rectifier $\mathrm{K}^{+}$-channel that slowly activates at membrane potentials at and below resting potential and slowly inactivates above resting potential.

\section{Responses to thalamic stimulation}

Electrical stimulation in DMA/DMP always evoked postsynaptic potentials in the recorded $\mathrm{MNH}$ neurons with latencies of $5 \mathrm{~ms}$ up to several $100 \mathrm{~ms}$. At resting membrane potential, the responses usually appeared as depolarizing PSPs.
When stimulation in DMA/DMP was strong enough to evoke action potentials in MNH neurons, typically 1 to 5 spikes superimposed to a long lasting depolarizing PSP occurred (Fig. 4A, B). In certain fast spiking neurons, thalamic stimulation induced a burst of action potentials with more than 500 ms duration (Fig. 4C).

When neurons were driven to different membrane potentials by intracellular current injection before and during afferent stimulation, we could study the reversal potentials of the evoked responses (Fig. 6). At more depolarized membrane potentials, the evoked responses appeared as a fast EPSP component with a reversal potential far more positive than the investigated range, and a slow, long lasting IPSP component reversing at about $65 \mathrm{mV}$. EPSPs that sometimes reached threshold could be superimposed to the slow IPSP.

\section{Pharmacology}

$N M D A$ : Sixteen $\mathrm{MNH}$ neurons were analyzed applying NMDA concentrations from 5 to $100 \mu \mathrm{M}$. In $75 \%$ of the neurons, the membrane potential broke down after a short period of enhanced spontaneous activity (Fig. 7A). In $12.5 \%$ of the neurons, the responses to thalamus stimulation were stronger (evoked EPSPs had higher amplitudes) during NMDA application. In one neuron $(6.25 \%)$, the spontaneous EPSPs were prolonged, and in another neuron $(6.25 \%)$, no change of spontaneous or evoked activity was observed, not even at high NMDA concentrations $(2.5 \mathrm{mM})$.

$A P V$ and Con-G: Five MNH neurons were analyzed with APV (50 to $200 \mu \mathrm{M}$ ) and five $\mathrm{MNH}$ neurons during Con-G $(50 \mu \mathrm{M})$ application. Both NMDA-antagonists affected EPSPs evoked by thalamus stimulations: In $60 \%$ of the tested neurons, APV reduced EPSP amplitudes by up to $55 \%$. Even with high APV concentrations $(2 \mathrm{mM})$, the evoked responses could not be completely blocked (Fig. 8). In $60 \%$ of the tested neurons, 


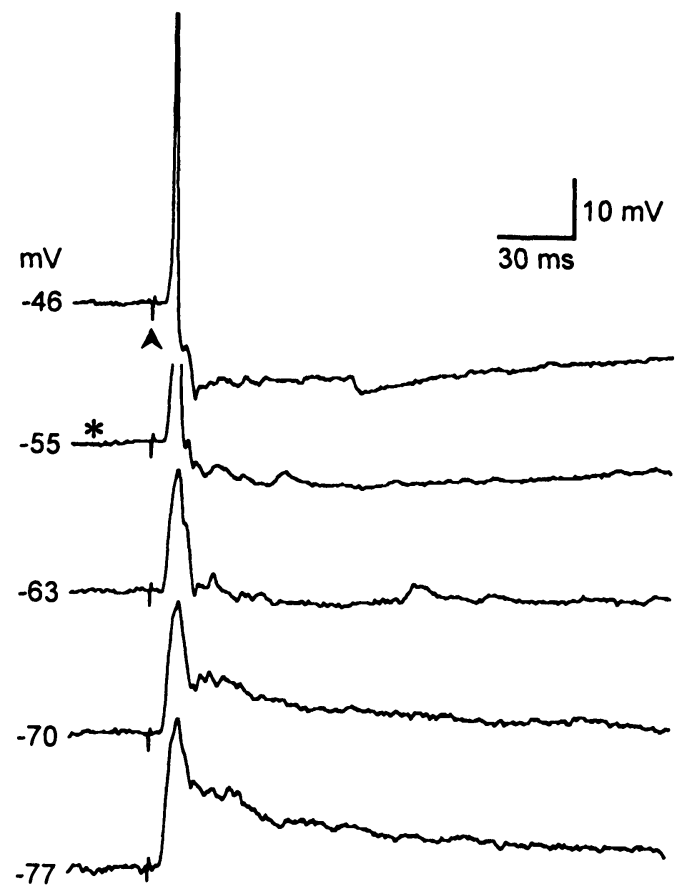

Fig. 6: Reversal potential of the slow IPSP component. Recording traces show the responses of an MNH neuron after single thalamus stimulation at different membrane potentials. The neuron's resting potential is indicated by an asterisk; the stimulus artifact is indicated by an arrowhead in the upper trace. The slow IPSP that is visible at more depolarized membrane potentials reverses at approx. $-65 \mathrm{mV}$.
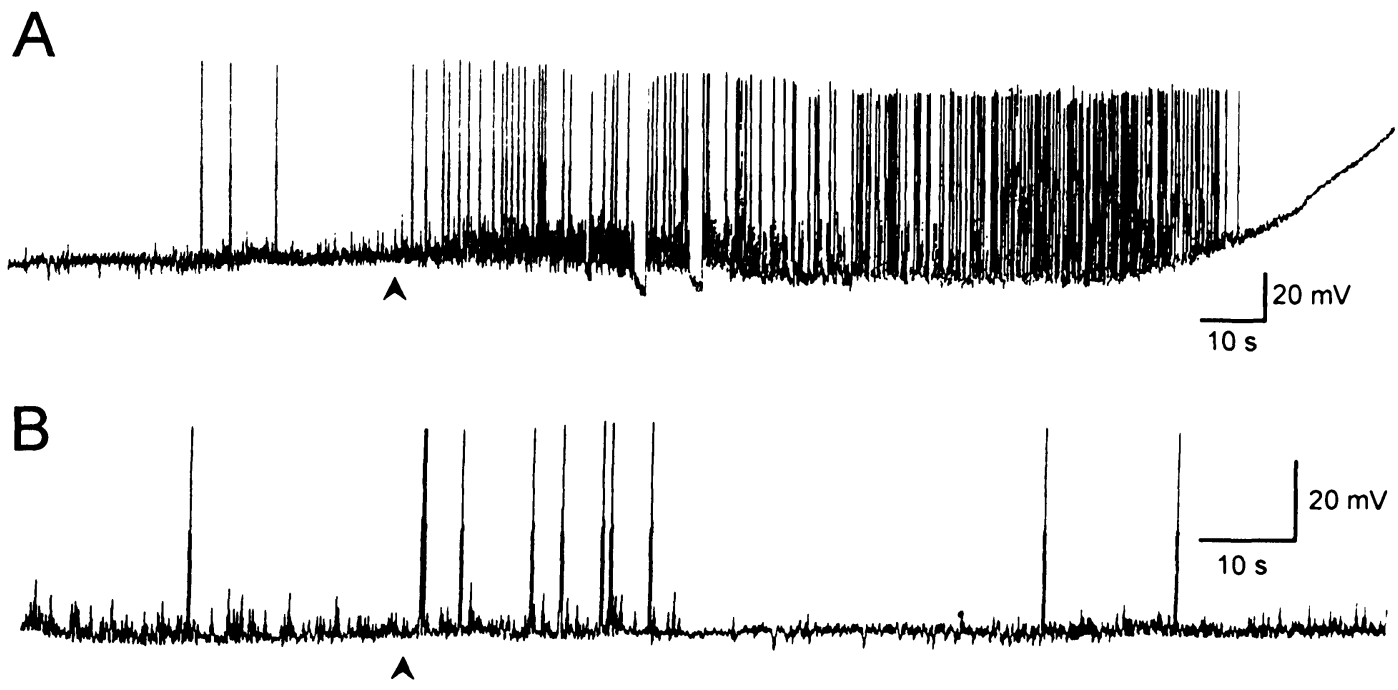

Fig. 7: Effect of pharmacological agents on the spontaneous activity of MNH neurons. The arrowheads indicate the time of bath application. A: NMDA ( $2 \mathrm{mM}$ final concentration) leads to an increase of spontaneous activity and finally the breakdown of the neuron's membrane potential. B: Bicuculline $(5 \mu \mathrm{M})$ reversibly changed the neuron's spontaneous activity. After an initial burst of action potentials, the number of spontaneous EPSPs decreased. 


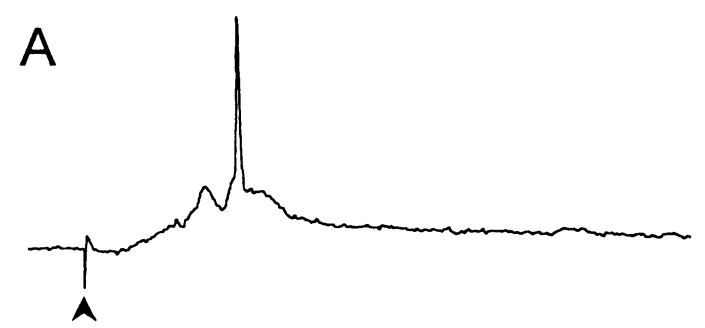

B
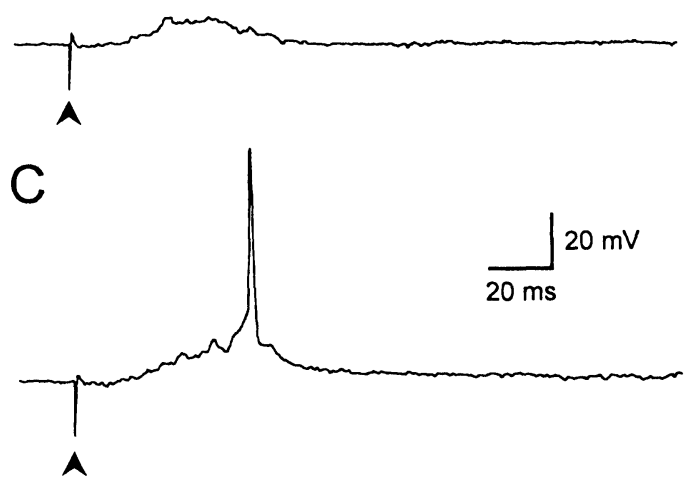

Fig. 8: APV attenuates the response of an MNH neuron to single thalamus stimulation. Stimulus artifacts are indicated by arrowheads. A: Control recording. B: $50 \mu \mathrm{M}$ APV (bath application). C: After 10 min wash.

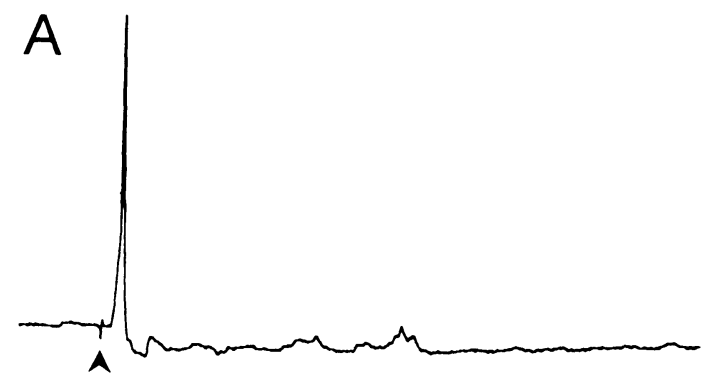

$B$

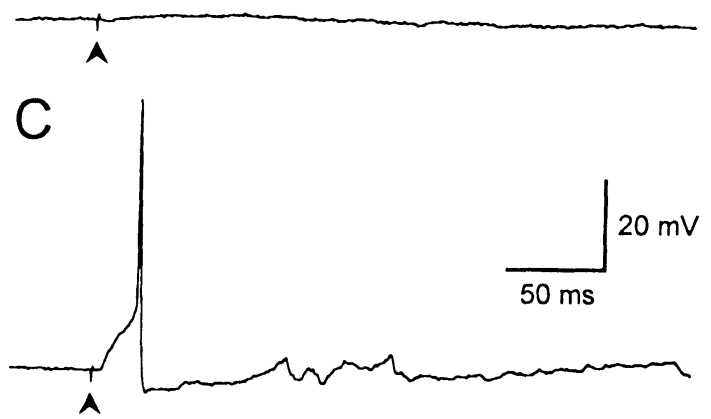

Fig. 9: CNQX blocks the response of an $\mathrm{MNH}$ neuron to single thalamus stimulation. Stimulus artifacts are indicated by arrowheads. A: Control recording. B: $10 \mu \mathrm{M} \mathrm{CNQX} \mathrm{(bath} \mathrm{application).} \mathrm{C:} \mathrm{After} 20$ min wash. 


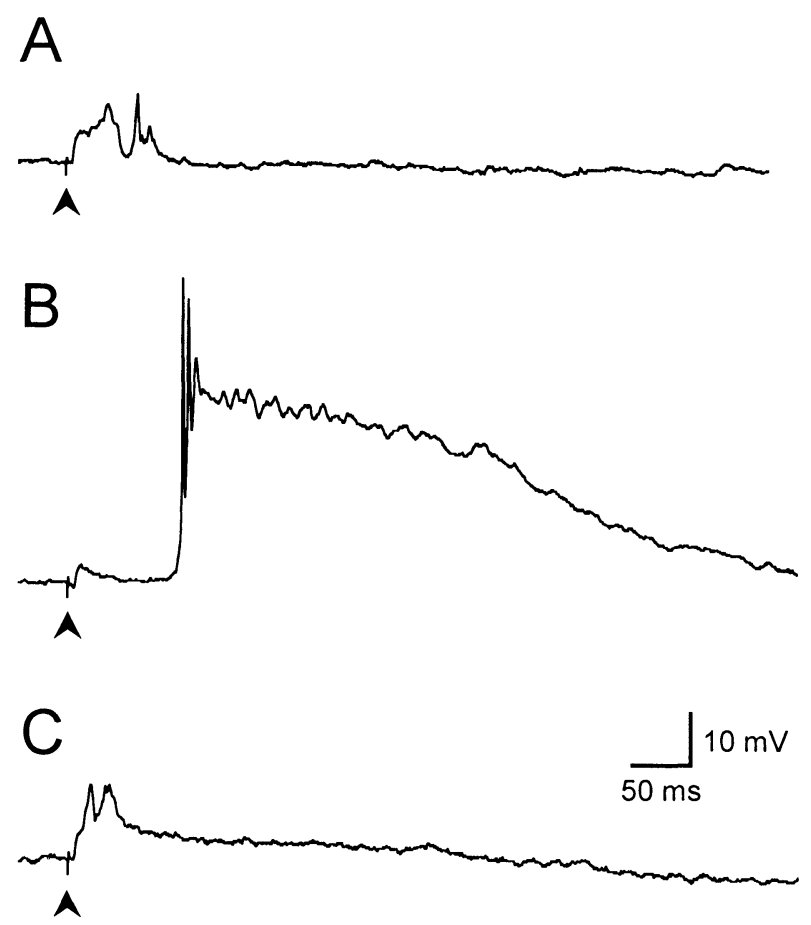

Fig. 10: Bicuculline changes the response of an MNH neuron to single thalamus stimulation. Stimulus artifacts are indicated by arrowheads. A: Control recording. B: $100 \mu \mathrm{M}$ bicuculline (bath application) blocks the slow PSP component while the fast PSP persists. An additional PSP with long latency and high amplitude appears. C: After 15 min wash.

Con-G reduced EPSP amplitudes by up to $80 \%$.

$C N Q X$ : With 5 to $10 \mu \mathrm{M}$ CNQX, the evoked responses disappeared completely in 10 of $12(83 \%)$ investigated neurons (Fig. 9), and excitatory spontaneous activity was no longer detectable. In two neurons $(17 \%)$, the EPSP amplitude was reduced by $70 \%$ and $40 \%$, respectively. Intracellular current injections during CNQX application confirmed that the neurons were in good condition and still capable of generating action potentials.

Bicuculline: The application of bicuculline methiodid enhanced spontaneous activity in all five neurons. Even low concentrations of $5 \mu \mathrm{M}$ had a short lasting effect (Fig. 7B). During thalamic stimulation, $100 \mu \mathrm{M}$ bicuculline methiodid suppressed the slow PSP component completely, whereas the fast PSP component remained (Fig. 10). Additionally, an enormous long-lasting depolarizing response with long latency occurred.

\section{Plasticity}

After thalamic high frequency stimulation, Seven of 32 neurons $(22 \%$; 4 regular spiking, 2 bursting, 1 fast spiking) showed an enhanced synaptic response to a test stimulus (Figs. 11, 12). We found an increase of the number of action potentials, integral and slope of EPSPs, as well as a decrease of the action potential latency (in one neuron the action potential latency increased). In two of these neurons $(6 \%$; 1 fast spiking, 1 regular spiking), the potentiation lasted longer than $15 \mathrm{~min}$. In five neurons (16\%; 3 regular spiking, 2 bursting), 

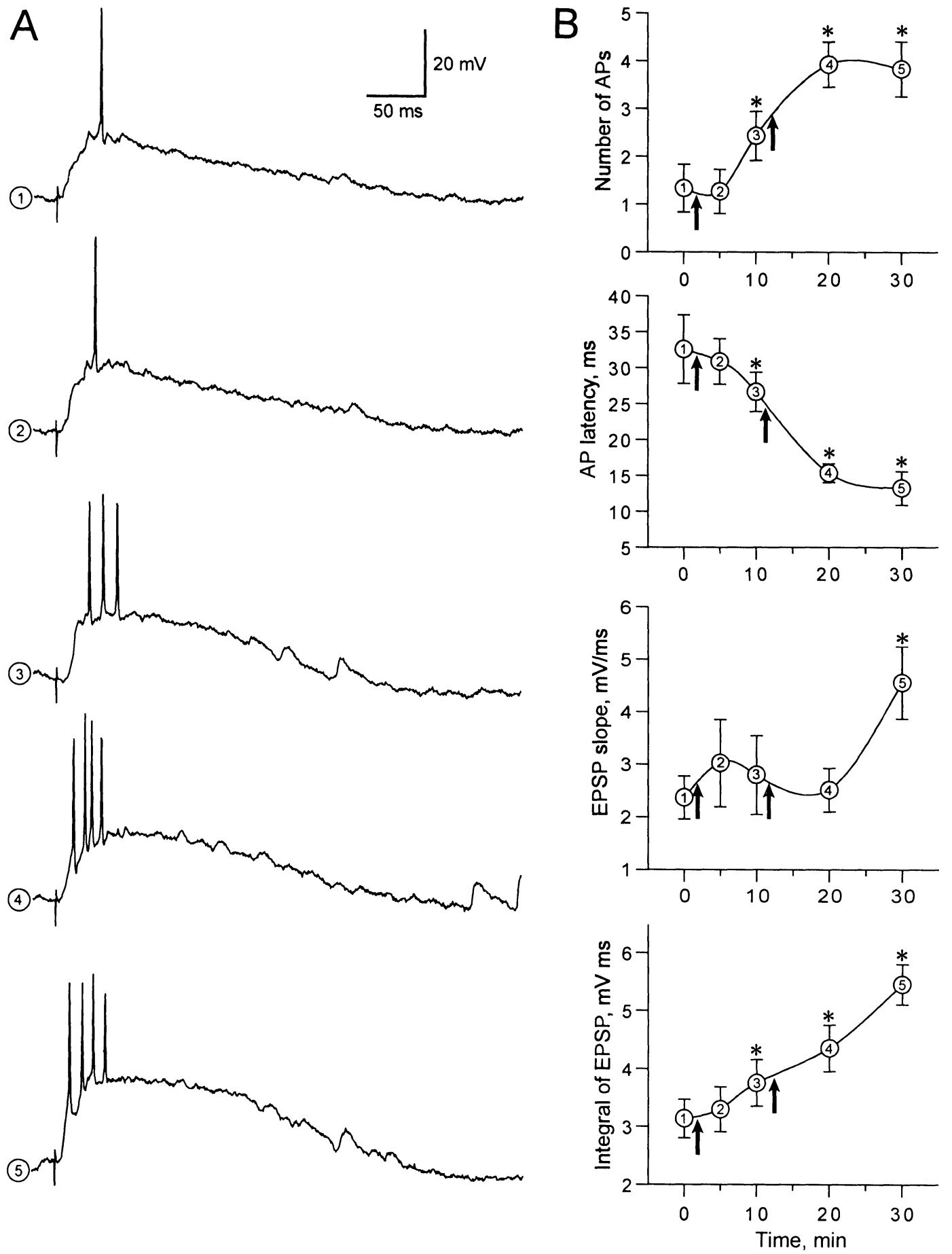

Fig. 11: Synaptic potentiation in an MNH neuron after two high frequency stimulation trains $(100 \mathrm{~Hz}, 1 \mathrm{~s}$ each) in the thalamus. A: Response to single thalamus stimulation; (1) control recording. (2) 4 min after first high frequency stimulation, (3) 9 min after first high frequency stimulation, (4) $9 \mathrm{~min}$ after second high frequency stimulation, (5) $19 \mathrm{~min}$ after second high frequency stimulation. B: Mean values \pm standard deviation of response parameters calculated out of 25 recordings each. Arrows indicate high frequency stimulation in the thalamus. Significant differences from $(1)(p<0.05)$ are indicated by asterisks. 

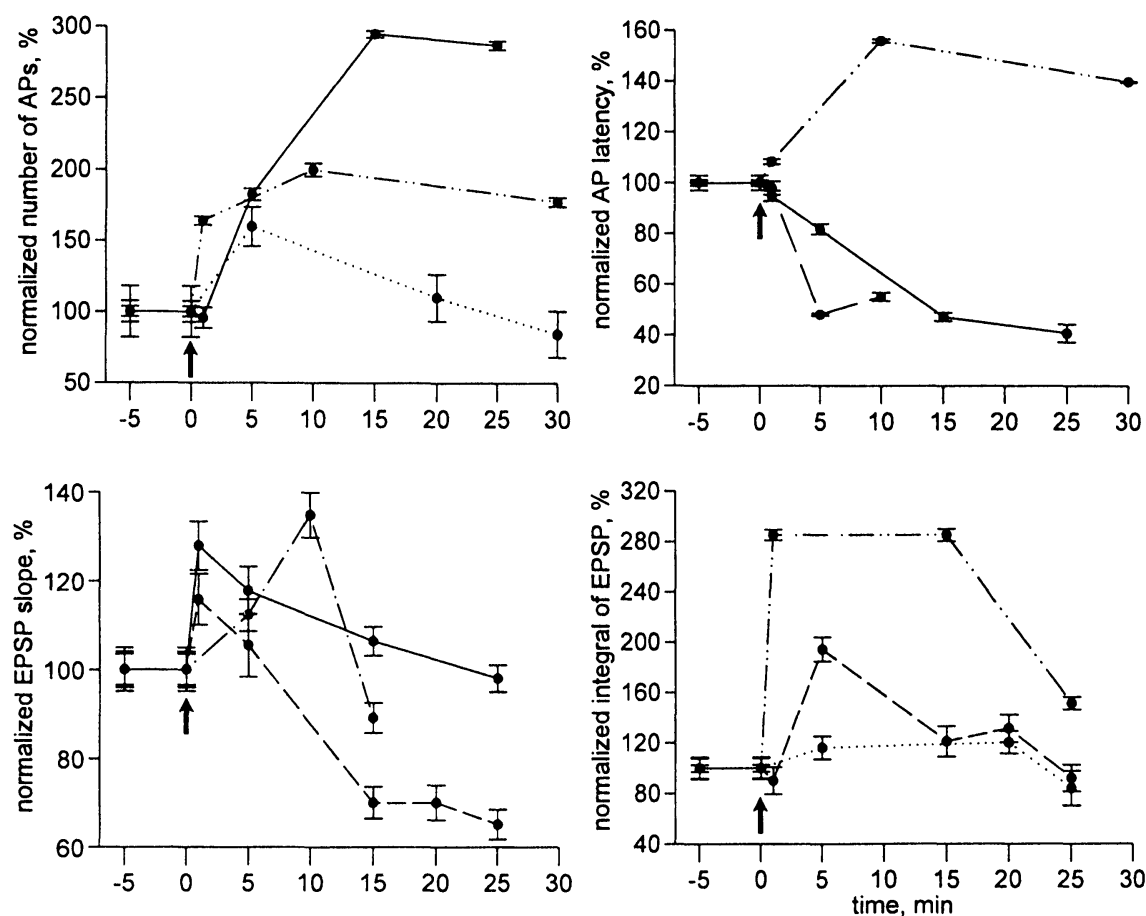

Fig. 12: Synaptic potentiation of individual MNH neurons (indicated by different line types) after high frequency thalamus stimulation. Mean values and standard errors of the mean were calculated out of 25 recordings, respectively. Arrows indicate high frequency stimulation.

the potentiation lasted only several minutes. Thalamic high frequency stimulation did not alter the resting potential.

\section{DISCUSSION}

\section{Physiological characterization of MNH-neurons in vitro}

The three physiologically defined neuron types (regular spiking, fast spiking, and bursting) that were found in $\mathrm{MNH}$ acute slice preparations (Wang, 1992) were also identified in MNH slice cultures, but with different percentages. Regular spiking neurons, the most abundant neuron type, were represented both in cultures $(75 \%)$ and in acute slices $(60 \%)$. The percentage of bursting neurons, however, was much lower in cultures $(12 \%)$ than in acute slices $(36 \%)$, whereas the percentage of fast spiking neurons was higher in cultures $(13 \%)$ than in acute slices $(4 \%)$. The different ratio of regular spiking neurons to bursting neurons in the tissue cultures could be due to a differential survival of these physiological neuron types under in vitro conditions. Alternatively, the surviving neurons might experience a change from bursting to regular or tonic mode, as described for guinea pig neocortical slices (Connors et al., 1982), in rat hippocampal slices (Scharfman, 1993), and in lobster stomatogastric ganglion cells (Turrigiano et al., 1994). We might have underestimated the occurrence of fast-spiking neurons both in vivo and in vitro because of the difficulty in obtaining stable recordings from this small neuron type. Brief recordings, during which 
the neurons were lost before stimulations could be started, point to a much larger percentage of fast spiking cells, similar to observations in rat neocortical explant cultures (Wolfson et al., 1989) and in neocortical slices (McCormick et al., 1985). Because MNH neurons are much larger in vitro than in vivo (Hofmann \& Braun, 1995), recording fast spiking neurons was easier than it might be in acute slices.

The time constant and AP duration were significantly shorter and the AP amplitude was significantly smaller in fast-spiking neurons than in regular spiking and bursting neurons. Particularly, short AP duration is a common feature of fastspiking neurons, described in MNH acute slices (Wang, 1992) as well as in various regions of mammalian cortex, where fast spiking neurons act as inhibitory interneurons (Connors \& Gutnick, 1990). A small AP amplitude and short time constant compared with other neuron types were also reported for fast spiking neurons in cocultures of rat cortex, striatum and substantia nigra (Plenz \& Kitai, 1998).

The presence of bursting, regular, and fast spiking neurons in $\mathrm{MNH}$-cultures indicates that the three principal neuronal spike patterns of $\mathrm{MNH}$ neurons persist in culture. Their physiological properties, such as time constant, input resistance, spike threshold, spike amplitude, and spike duration of MNH neurons remained stable over the first four weeks in vitro, which agrees with reports from other in vitro systems (Jourdain et al., 1996; Lohrke et al., 1998), paralleling the persistence of morphological parameters during this period (Hofmann \& Braun, 1995).

Thirty percent of the MNH-neurons showed inward rectification of hyperpolarizing electrotonic responses. Inward rectifier currents participate up to $50 \%$ in resting conductance of neurons with an inward rectifier potassium channel (Uchimura et al., 1989) and cause a robust resting potential, due to reduced input resistance and a shortened time constant at hyperpolarized levels (Nisenbaum \& Wilson, 1995). Thus, neurons are capable of introducing high thresholds for neuronal processing in a synaptic network. Inward rectification in the $\mathrm{MNH}$ has not been measured in vivo nor in acute slices.

Important measures for cell viability are resting potential, AP amplitude, and AP duration, which have been described for MNH acute slices (Wang, 1992). Cultured MNH neurons had mean resting potentials from $-61 \mathrm{mV}$ to $-68 \mathrm{mV}$ (depending on time in vitro), which is well within the range of resting potentials in $\mathrm{MNH}$ acute slices (from $-57 \mathrm{mV}$ to $-85 \mathrm{mV}$ ). Mean AP amplitudes were from $60 \mathrm{mV}$ to $66 \mathrm{mV}$ in cultures, somewhat lower than in $\mathrm{MNH}$ acute slices (range from $70 \mathrm{mV}$ to $100 \mathrm{mV}$ ), but still had a considerable overshoot (mean spike threshold was $-51 \mathrm{mV}$ to $-39 \mathrm{mV}$ ). Data about spike duration in acute slices are available only for fast-spiking neurons $(0.7 \mathrm{~ms})$, corresponding to values found in cultured $\mathrm{MNH}$.

As long as they lie within a 'normal' range, the input resistance, time constant, and spike threshold are measures for the excitability rather than the viability of a neuron. Cultured $\mathrm{MNH}$ neurons had input resistances ranging from $53 \mathrm{M} \Omega$ to $67 \mathrm{M} \Omega$ (depending on time in vitro), which is similar to values found in mammalian acute slices of cortex and hippocampus (Foehring et al., 1991; van Brederode \& Snyder, 1992; Scharfman, 1993; Beggs \& Kairiss, 1994). Additionally, the time constants of cultured MNH neurons ( $5 \mathrm{~ms}$ to $9 \mathrm{~ms}$ ) were similar to those in mammalian acute slices (Foehring et al., 1991; Scharfman, 1993). Whereas the input resistances and time constants were not measured in $\mathrm{MNH}$ acute slices, the AP thresholds ranged from $-58 \mathrm{mV}$ to $-50 \mathrm{mV}$. In $\mathrm{MNH}$ cultures, mean AP thresholds were -39 to $-51 \mathrm{mV}$, indicating that cultured neurons are less excitable than are acute slices. The spontaneous activity in MNH neurons demonstrates that some neurons in the coculture generate action potentials without external stimulation. Whether the spontaneously active neurons 
are located in the thalamus, in the $\mathrm{MNH}$, or in both parts of the culture, is not yet known. The occurrence of perforated synapses $(11 \%$ of the thalamus-MNH synapses) and of presynaptic mitochondria (39\% of the thalamus-MNH synapses), which theoretically reflect high synaptic activation and efficacy (Chang \& Greenough, 1984; Geinisman, 1993; Neuhoff et al., 1999), suggest that thalamus-MNH projections contribute to this spontaneous activity.

\section{Pharmacological characterization of synaptic connections in DMA/DMP-MNH-cocultures}

Our results confirm that thalamic axons grow into the MNH tissue and form functional synapses on MNH neurons. Most synapses appeared to be glutamatergic because CNQX, the AMPA/kainateantagonist, completely blocked synaptic responses in $\mathrm{MNH}$ neurons after the stimulation of thalamic fibers. That the slow IPSPs were also blocked indicates a glutamatergic input on inhibitory $\mathrm{MNH}$ neurons. In contrast, the observation that NMDA and the two NMDA antagonists, APV and Con-G, did not affect all tested neurons (NMDA 94\%; APV $60 \%$, Con-G 60\%) indicates that in certain neurons, the glutamatergic input is mediated solely through non-NMDA receptors.

By applying bicuculline to the cultures, we tested the presence of GABAergic neurotransmission in our culture system. Suppression of the slow PSP component of evoked responses by bicuculline demonstrates that GABAergic inhibition is represented in the intrinsic MNH network. As the fast PSP was not altered during bicuculline application, the thalamic synapses on $\mathrm{MNH}$ neurons are not likely to be GABAergic. The occurrence of a long lasting EPSP with high amplitude and long latency indicates that during the pharmacological blockade of GABAergic synapses, the excitatory neuronal activity was led back from $\mathrm{MNH}$ to the thalamus and reached the recorded MNH-neuron a second time. This phenomenon requires excitatory $\mathrm{MNH}$ neurons projecting back into DMA/DMP, which in fact were observed in the DiI-tracing experiments, although their transmitters are not yet known. Such a projection from MNH to DMA/DMP, however weak, does not develop in vivo (Wild, 1987; Metzger et al., 1996). The observed and hypothetical connections within DMA/DMP-MNH cocultures are summarized in Fig. 13.

\section{Ultrastructural characterization of thalamic synapses in DMA/DMP-MNH-cocultures}

The ultrastructural analysis of the Dil-labeled fibers revealed that $48 \%$ of thalamic synapses were asymmetric, $34 \%$ terminated on dendritic spines, but only $11 \%$ were both asymmetric and spine synapses. This result was surprising because the electrophysiological experiments demonstrated mainly glutamatergic projections from DMA/DMP to $\mathrm{MNH}$, and those synapses are reportedly asymmetric and located on dendritic spines (Peters et al., 1991). Perhaps the symmetric thalamic synapses in culture represent excitatory synapses without a clearly developed postsynaptic density, which was also described in mammalian cocultures (Distler \& Robertson, 1993). In contrast, we observed in vivo a strong correlation between Gray-type and dendritic localization in the $\mathrm{MNH}$ region: $85 \%$ to $98 \%$ (depending on cell type) of the spine synapses were found to be asymmetric (Faber, 1992). Nevertheless, in the in vivo study, no distinction was made among the synaptic subtypes with respect to their origin (as done in our in vitro analysis in which thalamic fibers were selectively labeled), so a direct comparison of the in vivo and in vitro synaptic characterization is not possible and requires further analysis.

In line with our previous findings that in vitro, the cell bodies and dendrites are larger than those in vivo (Hofmann \& Braun, 1995), synapses in 


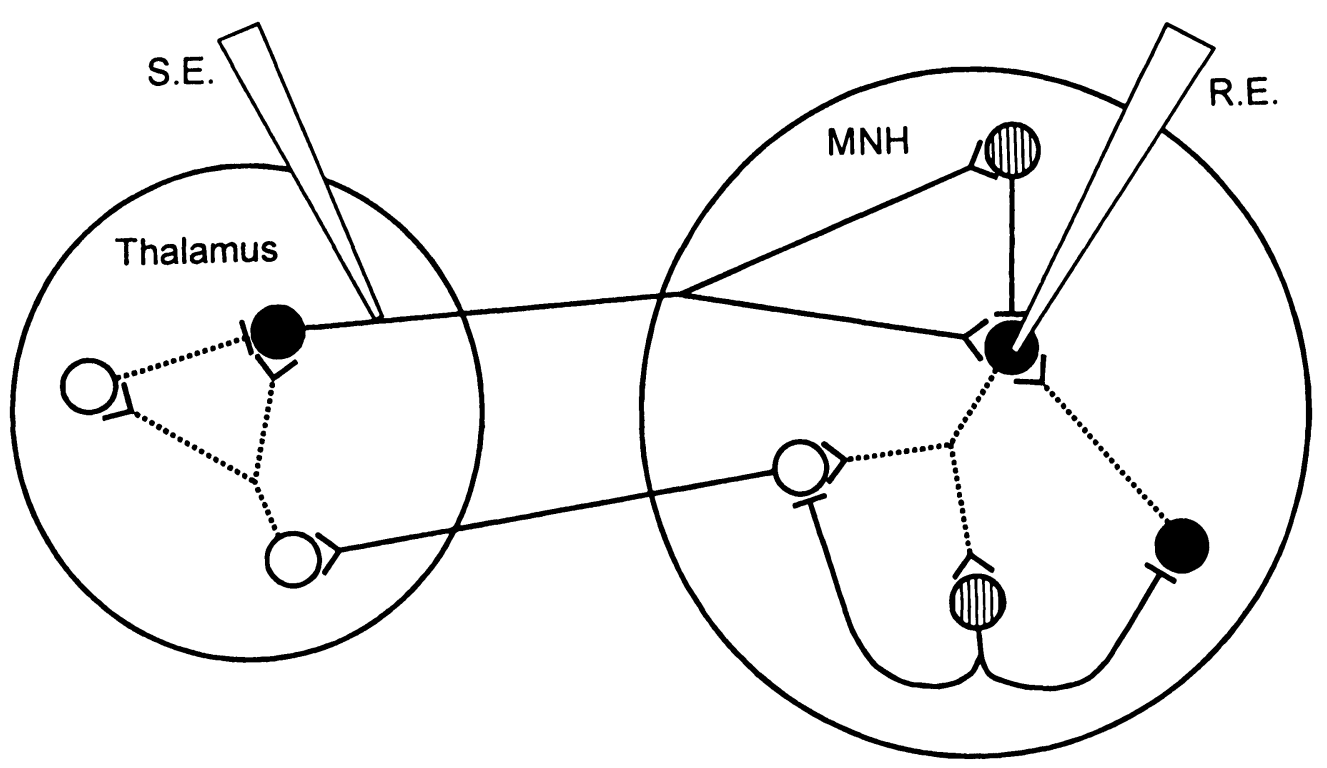

Fig. 13: Schematic drawing of putative synaptic connections in the thalamus-MNH-coculture. Black: glutamatergic neurons: hatched: GABAergic neurons; white: transmitter unknown. Black lines indicate connections supported by this study, dotted lines indicate hypothetical projections. S.E.: stimulation electrode; R.E.: recording electrode.

culture also appear much larger than in vivo. Spine synapses in vivo displayed mean PSD lengths ranging from $0.21 \mu \mathrm{m}$ to $0.31 \mu \mathrm{m}$ (depending on cell type), whereas in vitro thalamic spine synapses had a mean PSD length of $0.60 \mu \mathrm{m}$. A similar synaptic enlargement occurred in PSDs of shaft synapses, which in vivo were $0.23 \mu \mathrm{m}$ to $0.46 \mu \mathrm{m}$ long (Faber, 1992) and around $0.87 \mu \mathrm{m}$ in vitro. Synaptic enlargement was also reflected by the size of the mean presynaptic areas, which ranged for spine synapses from $0.06 \mu \mathrm{m}^{2}$ to $0.16 \mu \mathrm{m}^{2}$ (depending on cell type) in vivo and $1.41 \mu \mathrm{m}^{2}$ in vitro, and for shaft synapses from $0.13 \mu \mathrm{m}^{2}$ to $0.22 \mu \mathrm{m}^{2}$ in vivo and $1.14 \mu \mathrm{m}^{2}$ in vitro. Again, directly comparing these observations in vivo and in vitro is difficult because of methodological inadequacies; for example, different shrinkage factors, caused by different fixation and embedding protocols, or different sampling procedures may at least be partly responsible for the discrepancies.

\section{Synaptic plasticity}

Synaptic potentiation by high-frequency stimulation of the DMA/DMP tissue was inducible in $22 \%$ of the MNH neurons in culture, as opposed to $60 \%$ responsive neurons reported for acute slice preparations (Wang et al., 1994). The physiological changes occurring after tetanic stimulation in vitro were similar to those in $\mathrm{MNH}$ acute slices (Wang, 1992; Wang et al., 1994) and in slices from another learning-relevant forebrain area (Bradley et al., 1991; Matsushima \& Aoki, 1995). As in slice preparations, we found an increase of the number of action potentials, integral and slope of EPSPs. and a decrease of the action potential latency. On 
the other hand, differences of cellular responses to tetanic stimulation also became obvious. For instance, in our coculture system we did not see the large depolarization (from resting levels to about $-30 \mathrm{mV}$, lasting about $1 \mathrm{~min}$ ), which occurred in $36 \%$ of $\mathrm{MNH}$ neurons after tetanus and preceded the LTP phase in acute slice preparations. This result might be one explanation for the reduced percentage of potentiated neurons in cultures. The differences between tetanization effects in MNH acute slices and DMA/DMP-MNH cocultures might be related, to a large extent, to the different anatomical features. Whereas in DMA/DMP-MNH cocultures the stimulation electrode was placed in the DMA/DMP partnamely, stimulation was almost purely thalamic-in $\mathrm{MNH}$ slices the stimulation electrode was placed on fiber bundles traversing through the lobus parolfactorius. The latter not only contains the DMA/DMP afferents but also includes afferents from the caudal neostriatum (Metzger et al., 1998) and probably mesencephalic dopaminergic, and serotonergic afferents as well.

In summary, our morphological and physiological characterization of DMA/DMP-MNH cocultures revealed that this avian in vitro system provides a suitable model for physiological and pharmacological studies on synaptic plasticity under highly controlled conditions.

\section{REFERENCES}

Beggs JM, Kairiss EW. Electrophysiology and morphology of neurons in rat perirhinal cortex. Brain Res 1994; 665: 18-32.

Bischof HJ, Rollenhagen A. Behavioural and neurophysiological aspects of sexual imprinting in zebra finches. Behav Brain Res 1999; 98: 267-276.

Bock J, Braun K. Differential emotional experience leads to pruning of dendritic spines in the forebrain of domestic chicks. Neural Plast 1998; 6: 17-27.

Bock J, Braun K. Blockade of N-methyl-D-aspartate receptor activation suppresses learning-induced synaptic elimination. Proc Natl Acad Sci USA 1999; 96: 2485-2490.

Bock J, Schnabel R, Braun K. Role of the dorso-caudal neostriatum in filial imprinting of the domestic chick: A pharmacological and autoradiographical approach focused on the involvement of NMDAreceptors. Eur J Neurosci 1997; 9: 1262-1272.

Bock J, Wolf A, Braun K. Influence of the N-methyl-Daspartate receptor antagonist DL-2-amino-5-phosphonovaleric acid on auditory filial imprinting in the domestic chick, Neurobiol Learn Mem 1996; 65: 177-188.

Bolhuis JJ, Honey RC. Imprinting, learning and development: from behaviour to brain and back. Trends Neurosci 1998; 21:306-311.

Bradley PM, Burns BD, Webb AC. Potentiation of synaptic responses in slices from the chick forebrain. Proc Royal Soc London Series B- Biol Sci 1991; 243: 19-24.

Braun K, Bock J, Metzger M, Jiang S, Schnabel R. The dorsocaudal neostriatum of the domestic chick: A structure serving higher associative functions. Behav Brain Res 1999; 98: 211-218.

Bredenkötter $M$, Braun $K$. Changes of neuronal responsiveness in the mediorostral neostriatum/ hyperstriatum after auditory filial imprinting in the domestic chick. Neuroscience 1997; 76: 355-365.

Bredenkötter M, Braun K. Development of neuronal responsiveness in the mediorostral neostriatum/ hyperstriatum ventrale during auditory filial imprinting in domestic chicks. Neurobiol Learning Mem 2000; 73: 114-126.

van Brederode JFM, Snyder GL. A comparison of electrophysiological properties of morphologically identified cells in layers $5 \mathrm{~B}$ and 6 of the rat neocortex. Neuroscience 1992; 50: 315-337.

Chang FL, Greenough WT. Transient and enduring morphological correlates of synaptic activity and efficacy change in the rat hippocampal slice. Brain Res 1984; 309: 35-46.

Connors BW, Gutnick MJ. Intrinsic firing patterns of diverse neocortical neurons. Trends Neurosci 1990; 13: 99-104.

Connors BW, Gutnick MJ, Prince DA. Electrophy'siological properties of neocortical neurons in vitro. $\mathrm{J}$ Neurophysiol 1982; 48: 1302-1320.

DeCasper AJ, Fifer WP. Of human bonding: newborns prefer their mother's voices. Science $1980 ; 208$ : 1174-1176. 
Distler PG, Robertson RT. Formation of synapses between basal forebrain afferents and cerebral cortex neurons: An electron microscopic study in organotypic slice cultures. J Neurocytol 1993; 22: 627-643.

Faber H. Ultrastrukturelle Konsequenzen des akustischen Prägungslernens auf identifizierte Neuronentypen im Telencephalon von Haushuhnküken (Gallus gallus domesticus). PhD thesis, Technical University of Darmstadt, Germany, 1992.

Foehring RC, Lorenzon NM, Herron P, Wilson CJ. Correlation of physiologically and morphologically identified neuronal types in human association cortex in vitro. J Neurophysiol 1991; 66: 1825-1837.

Geinisman Y. Perforated axospinous synapses with multiple, completely partitioned transmission zones: Probable structural intermediates in synaptic plasticity. Hippocampus 1993; 3: 417-434.

Griffiths D, Dickinson A, Clayton N. Episodic memory: What can animals remember about their past? Trends Cogn Sci 3; 1999: 74-80.

Gruss M, Braun K. Stimulus-evoked increase of glutamate in the mediorostral neostriatum/ hyperstriatum ventrale of domestic chick after auditory filial imprinting: An in vivo microdialysis study. $J$ Neurochem 1996; 66: 1167-1173.

Hofmann H, Braun K. Slice cultures of the imprintingrelevant forebrain area $\mathrm{MNH}$ of the domestic chick: Quantitative characterization of neuronal morphology. Develop Brain Res 1995; 86: 283-296.

Horn G. Neural bases of recognition memory investigated through an analysis of imprinting. Philosoph Trans Royal Soc London Series B-Biol Sci 1990; 329: 133-134.

Jourdain P, Poulain DA, Theodosis DT, Israel JM. Electrical properties of oxytocin neurons in organotypic cultures from postnatal rat hypothalamus. J Neurophys 1996; 76: 2772-2785.

Lohrke S, Kungel M, Friauf E. Electrical membrane properties of trapezoid body neurons in the rat auditory brain stem are preserved in organotypic slice cultures. J Neurobiol 1998; 36: 395-409.

Maier V, Scheich $\mathrm{H}$. Acoustic imprinting leads to differential 2-deoxy-D-glucose uptake in the chick forebrain. Proc Natl Acad Sci USA 1983; 80: $3860-3864$.

Matsushima T, Aoki K. Potentiation and depotentiation of DNQX-sensitive fast excitatory synaptic transmission in telencephalon of the quail chick. Neurosci Lett 1995; 185: 179-182.
McCormick DA, Connors BW, Lighthall JW, Prince DA. Comparative electrophysiology of pyramidal and sparsely spiny stellate neurons of the neocortex. J Neurophysiol 1985; 54: 782-806.

Metzger M, Jiang S, Wang J, Braun K. Organization of the dopaminergic innervation of forebrain areas relevant to learning: A combined immunohistochemical/retrograde tracing study in the domestic chick. J Comp Neurol 1996; 376: 1-27.

Metzger M, Jiang S, Braun K. Organization of the dorsocaudal neostriatal complex: A retrograde and anterograde tracing study in the domestic chick with special emphasis on pathways relevant to imprinting. J Comp Neur 1998; 395: 380-404.

Mooney R. Sensitive periods and circuits for learned birdsong. Curr Opin Neurobiol 1999; 9: 121-127.

Neuhoff H, Roeper J, Schweizer M. Activity-dependent formation of perforated synapses in cultured hippocampal neurons. Eur J Neurosci 1999; 11: 42414250 .

Nisenbaum ES, Wilson CJ. Potassium currents responsible for inward and outward rectification in rat neostriatal spiny projection neurons. J Neurosci 1995; 15: 4449-4463.

Peters A, Palay SL, Webster HF. The fine structure of the nervous system. Oxford University Press, 1991.

Plenz D, Kitai ST. Up and down states in striatal medium spiny neurons simultaneously recorded with spontaneous activity in fast-spiking interneurons studied in cortex-striatum-substantia nigra organotypic cultures. J Neurosci 1998; 18: 266-283.

Rose SPR. God's organism? The chick as a model system for memory studies. Learn Mem 2000; 7 : $1-17$.

Scharfman HE. Spiny neurons of area CA3c in rat hippocampal slices have similar electrophysiological characteristics and synaptic responses despite morphological variation. Hippocampus 1993; 3: 9-28.

Scheich H, Wallhäußer-Franke E, Braun K. Does synaptic selection explain auditory imprinting? In: Squire LR, Weinberger NM, Lynch G, McGaugh JL, eds, Memory: Organization and locus of change, Oxford University Press, 1991; 114-159.

Tramontin AD, Brenowitz EA. Seasonal plasticity in the adult brain. Trends Neurosci 2000; 23: 251-258.

Turrigiano G, Abbott LF, Marder E. Activity-dependent changes in the intrinsic properties of cultured neurons. Science 1994; 264: 974-977.

Uchimura N, Cherubini E, North RA. Inward rectifica- 
tion in rat nucleus accumbens neurons. J Neurophys 1989; 62: 1280-1286.

Wallhäußer E, Scheich H. Auditory imprinting leads to differential 2-deoxyglucose uptake and dendritic spine loss in the chick rostral forebrain. Brain Res 1987; 428: 29-44.

Wang $X$. Elektrophysiologische Eigenschaften und Langzeitpotenzierung in einem gedächtnisrelevanten Vorderhirngebiet des Haushuhnkükens in vitro. $\mathrm{PhD}$ thesis, Technical University Darmstadt, 1992.
Wang X, Babinsky R, Scheich H. Synaptic potentiation and depression in slices of mediorostral neostriatum-hyperstriatum complex, an auditory imprintingrelevant area in chick forebrain. Neuroscience 1994; 60: 689-699.

Wild JM. Thalamic projections to the paleostriatum and neostriatum in the pigeon (Columba liva). Neuroscience 1987; 20: 305-327.

Wolfson B, Gutnick MJ, Baldino F. Jr. Electrophysiological characteristics of neurons in neocortical explant cultures. Exp Brain Res 1989; 76: 122-130. 

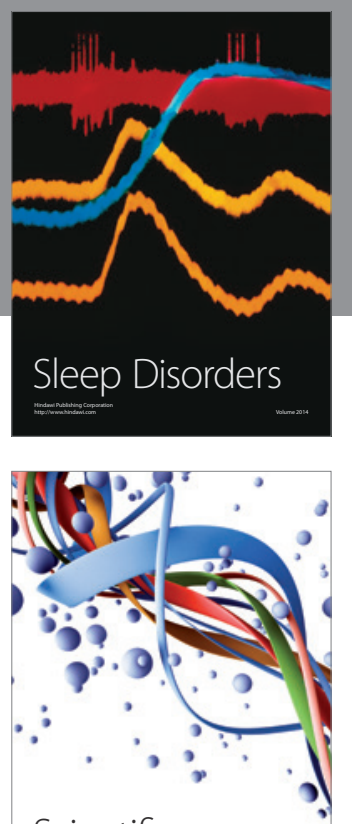

Scientifica
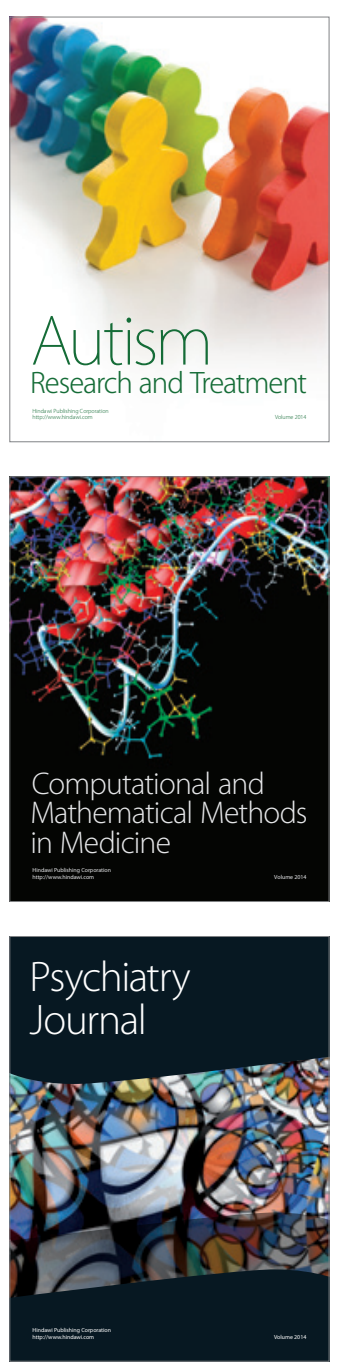
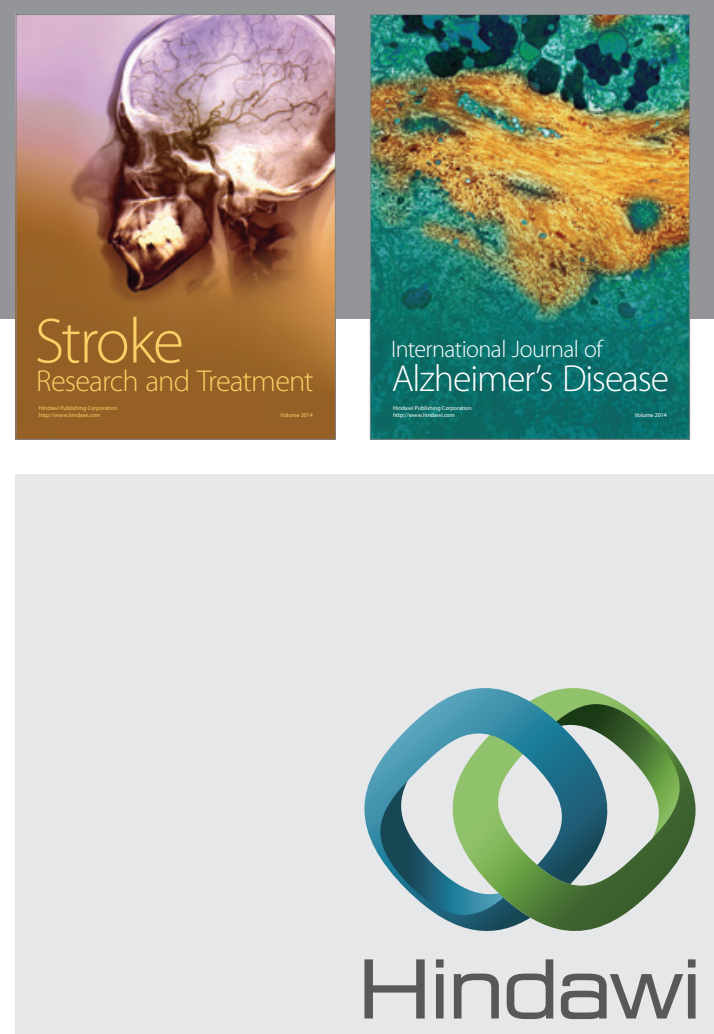

Submit your manuscripts at

http://www.hindawi.com
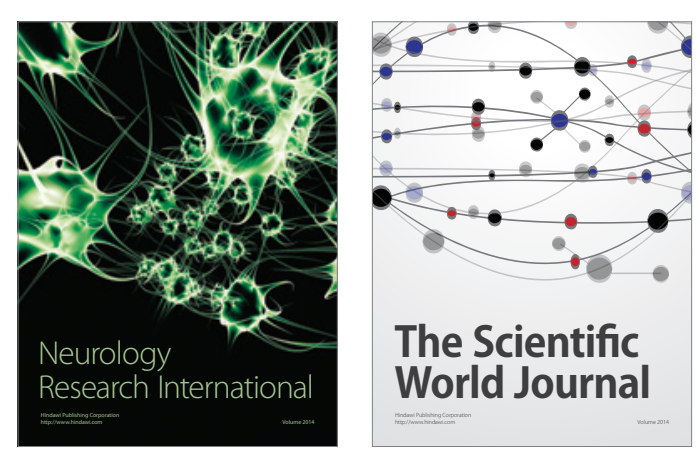

The Scientific World Journal

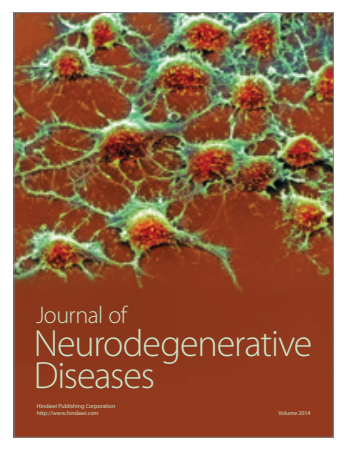

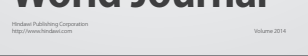

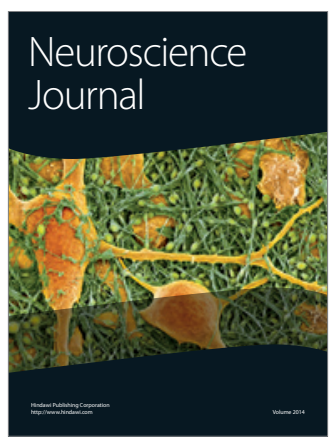

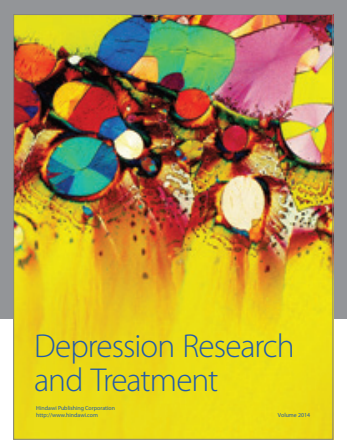
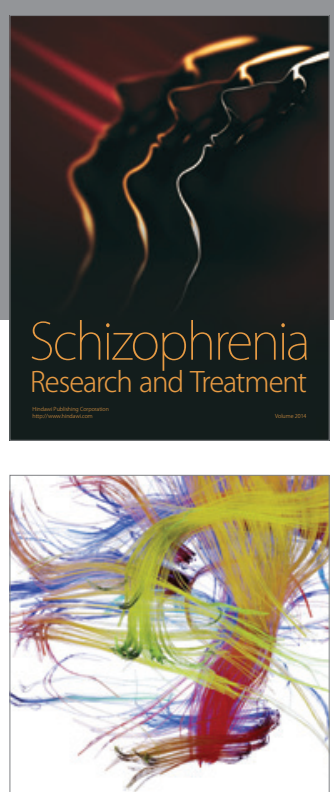

Brain Science

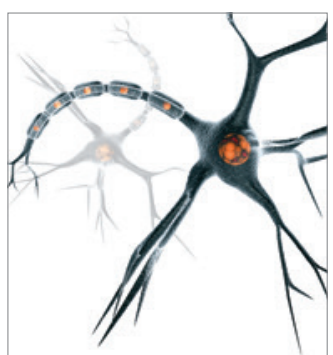

Neural Plasticity
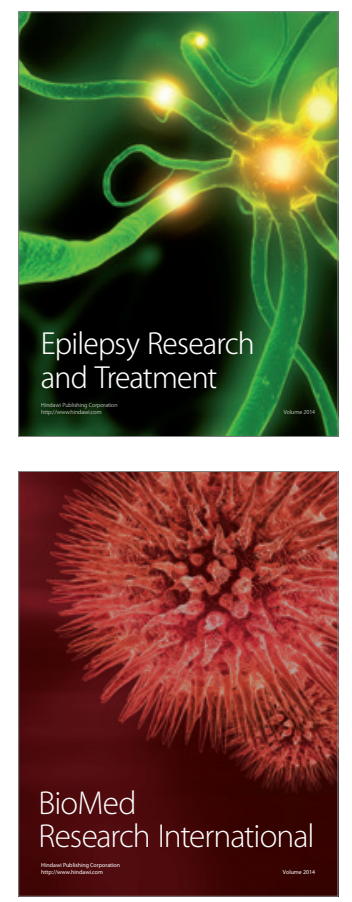

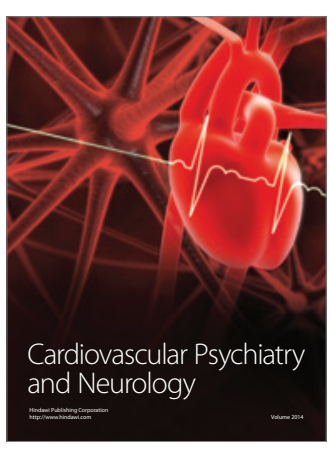

Parkinson's

Disease
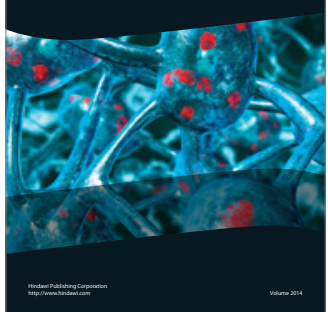